\title{
ROCK MASS RATING OF CAVITY LIMESTONE LAYER IN REMBANG, CENTRAL JAVA, INDONESIA
}

\author{
R. Andy Erwin Wijaya*1, Dwikorita Karnawati ${ }^{2}$ Srijono $^{2}$, and Wahyu Wilopo ${ }^{2}$ \\ ${ }^{1}$ Department of Mining Engineering, Sekolah Tinggi Teknologi Nasional, Indonesia \\ ${ }^{2}$ Department of Geological Engineering, Faculty of Engineering, Universitas Gadjah Mada, Indonesia
}

\begin{abstract}
Safety of a limestone mining activity requires a good mine design. Mine design is determined by the rock mass quality, which varies from one mine location to another, depending on the geological conditions. The research area is located in limestone quarry of Sale District, Rembang Regency, Central Java Province, Indonesia. In the study area, a cavity zone is exposed at the wall of quarry bench and occurs by a solution process. The cavity layer zone is a weak zone which has caused bench failures. The objective of this research is to evaluate the quality of the cavity limestone layer for a safe mine design using Rock Mass Rating (RMR) system. Final result of the research is a rock mass characterization, specifically for the cavity limestone layer.
\end{abstract}

Keywords: Rock mass rating, limestone, cavity layer

\section{Introduction}

Limestone mining in Sale District, Rembang Regency, Center Java Province-Indonesia uses the quarry method, which is one of the surface mine methods (Hustrulid and Kuchta, 1995). Reliable estimates of the strength and deformation characteristics of rock masses are required for almost any form of analysis used for the mine design of surface excavations specifically for bench of limestone quarry. Rock mass classi-

${ }^{*}$ Corresponding author: R.A.E. WIJAYA, Department of Mining Engineering, Sekolah Tinggi Teknologi Nasional, Indonesia. E-mail: andy_sttnas@yahoo.com fication systems used to be of prime importance to estimate rock mass behavior. In $1973 \mathrm{Bi}$ eniawski was the first who developed the rock mass rating (RMR) system (CSIR known as the South African Council of Scientific and Industrial Resarch), and sustained its development until 1989. By the available data its versions found more than 350 applications in underground opening, tunnels, underground mines, and open-pit slope designs. The RMR system is used in this research to determine rock mass strength of the surface mine, particularly the limestone quarry. Some parameters are required in the RMR analysis. The main parameters are strength of intact rock, RQD, spacing of discontinuity, condition of discontinuity and groundwater (Table 1).

\section{Location and site characterization}

The research area is located in limestone quarry of Sale District, Rembang Regency, Central Java Province, Indonesia. The research area is bounded by coordinates: $558000 \mathrm{mE}-558600$ $\mathrm{mE}$ and $9240400 \mathrm{mN}-9241000 \mathrm{mN}$ (Figure 1).

\section{Geological condition}

Rembang Zone is part of the Northern East Java Basin that runs from Tuban eastwards through Lamongan, Gresik, and almost the entire island of Madura. The morphology of the Rembang Zone can be divided into three morphological units, namely lowland, undulating hills, 
Table 1: Rock mass rating system (Bieniawski, 1989).

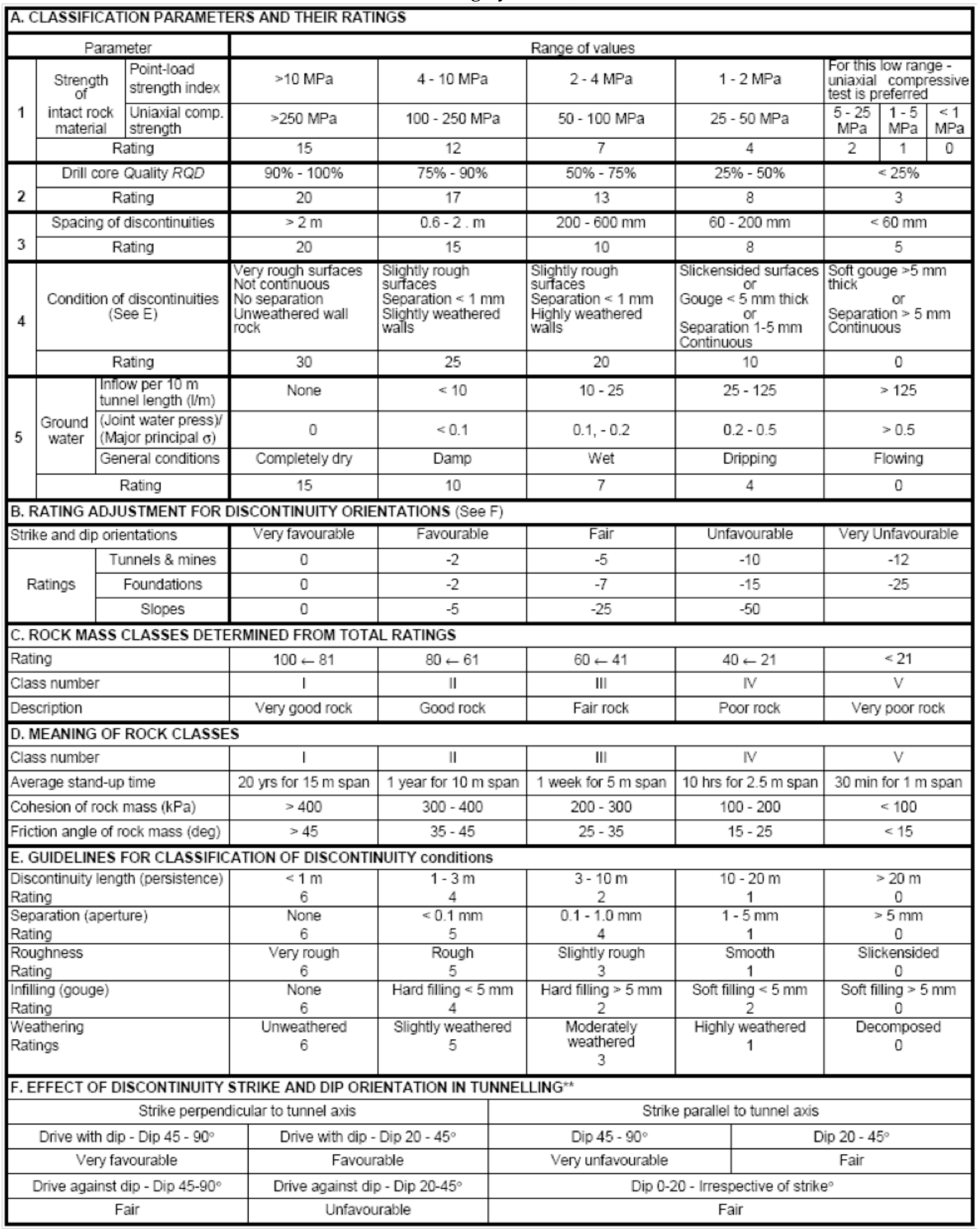




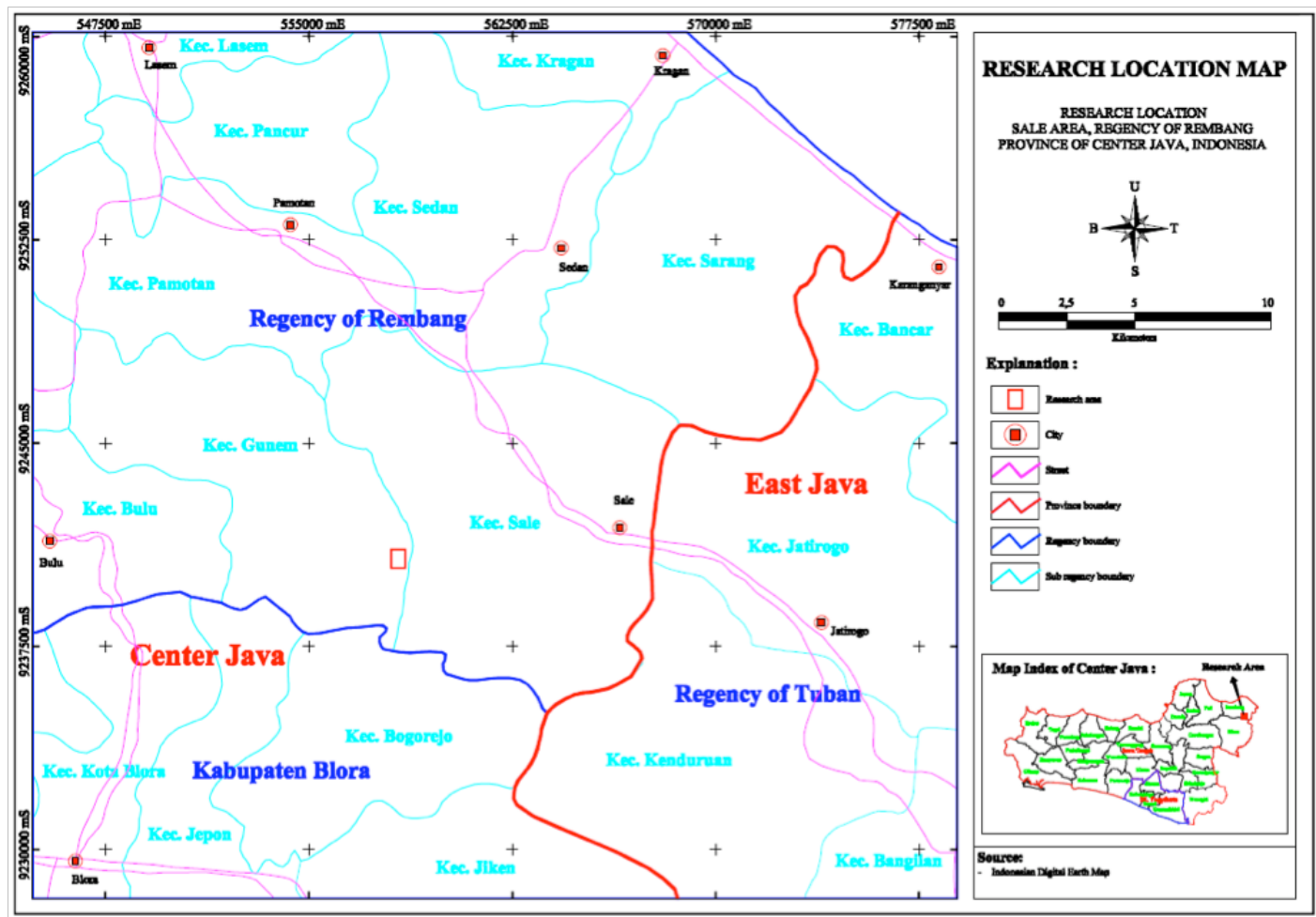

Figure 1: Location of the research area (red box).

and steep hills. Based on the geological map of Jatirogo (Figure 2), the research area is covered by Paciran Formation, which consists of massive limestone and dolomitic limestone (Situmorang, et al., 1992). The rocks in the Rembang Zone have undergone intensive folding and faulting resulting in anticlines and synclines.

\section{Solution cavity layer in limestone quarry}

In some parts of the limestone quarry area, there is cavity zone, which is formed by solution of limestone, at the wall of quarry bench (Figure 3). The cavity zone is a potentially weak zone of bench failure. The cavity zone is from 50 to $300 \mathrm{~cm}$ wide and located at an elevation of approximately $389 \mathrm{~m}$ above sea level. The slope of quarry bench ranges from $60^{\circ}$ to $75^{\circ}$. Observation of limestone cavity zone was conducted at 10 location points with A to J sample names (Figure 4). Location of each outcrop observation point in coordinates as follow (Table 2 ) and the cavity layer map (see Figure 5).
Table 2: Coordinates of cavity limestone layer outcrop observation.

\begin{tabular}{ccc}
\hline Sampel Code & $\mathbf{m E}$ & $\mathbf{m N}$ \\
\hline A & 558358 & 9240927 \\
\hline B & 558383 & 9240925 \\
\hline C & 558404 & 9240928 \\
\hline D & 558419 & 9240931 \\
\hline E & 558433 & 9240936 \\
\hline F & 558444 & 9240942 \\
\hline G & 558459 & 9240952 \\
\hline H & 558473 & 9240963 \\
\hline I & 558487 & 9240975 \\
\hline J & 558497 & 9240988 \\
\hline
\end{tabular}


WIJAYA et al.

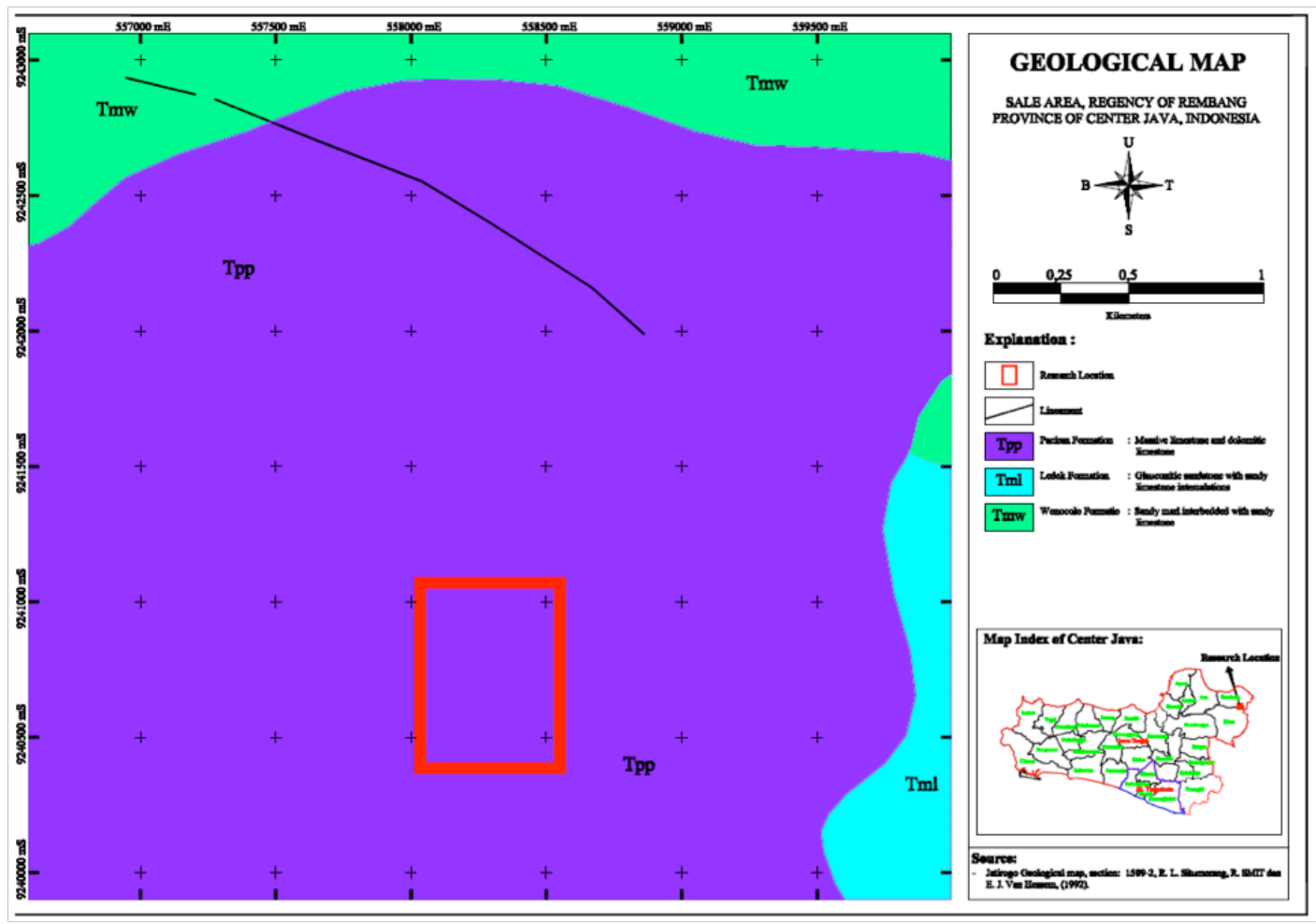

Figure 2: Geological map.

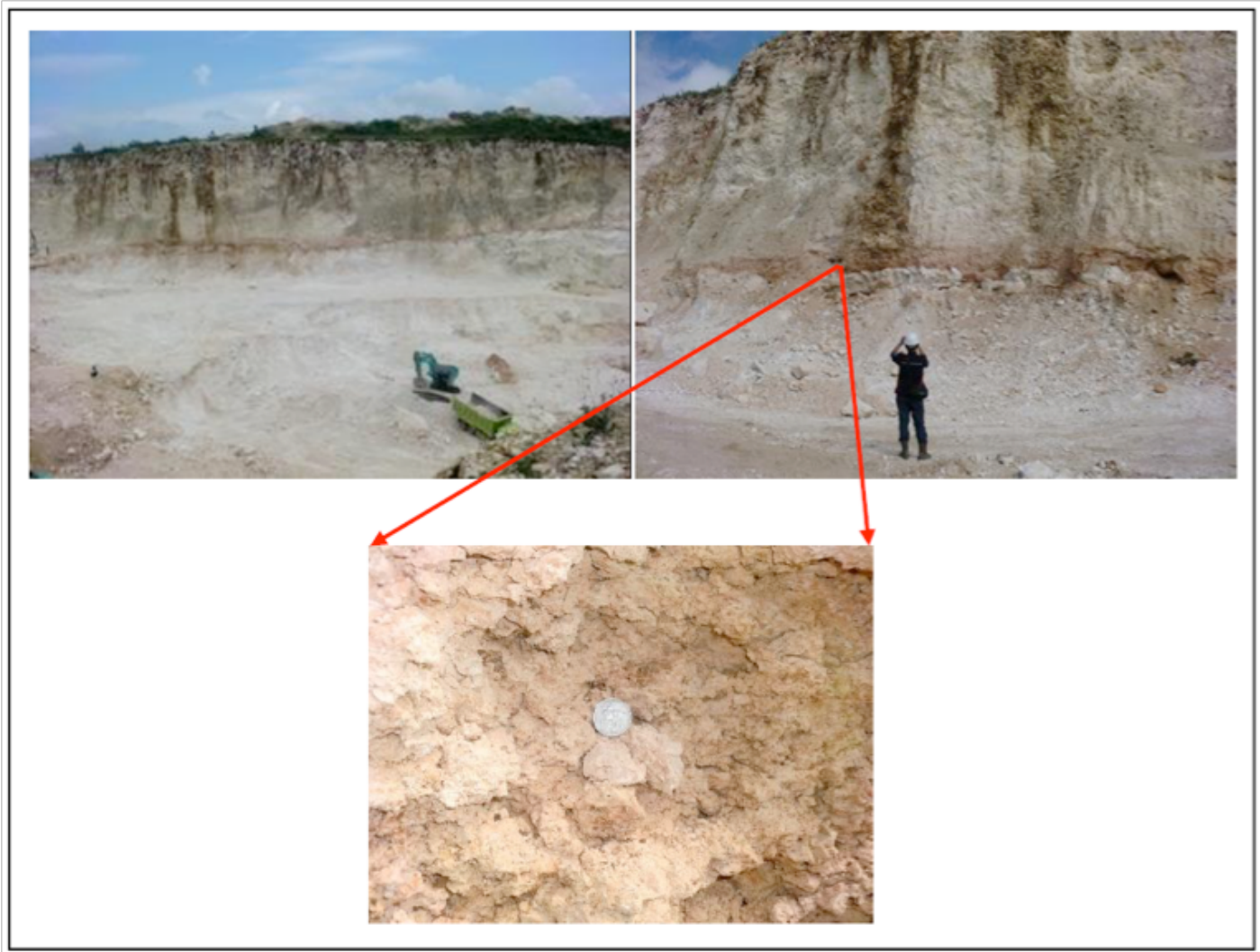

Figure 3: Cavity layer condition in limestone quarry. 
ROCK MASS RATING OF CAVITY LIMESTONE LAYER IN REMBANG, CENTRAL JAVA
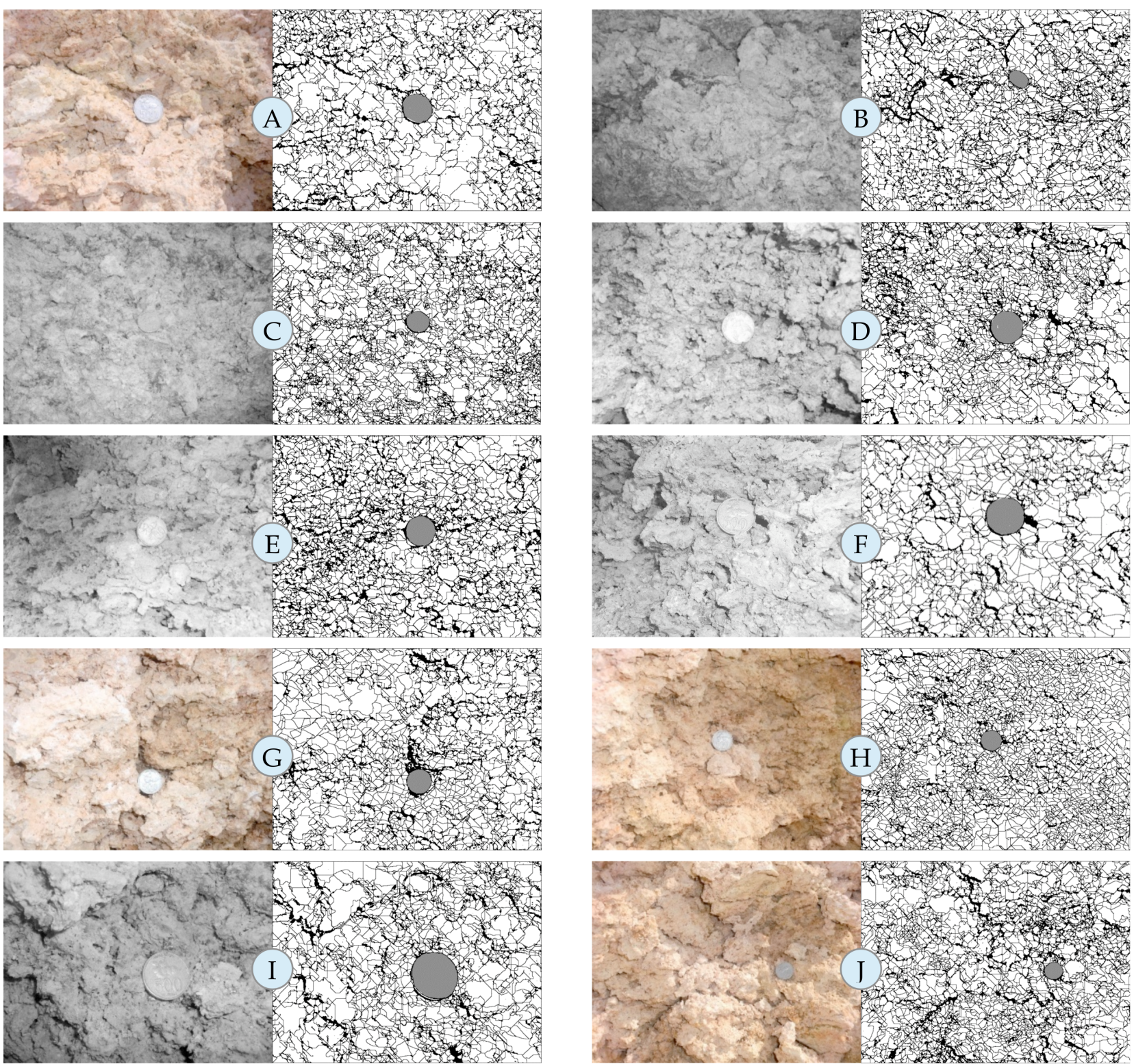

Figure 4: Limestone cavity layer outcrop. 
WIJAYA et al.

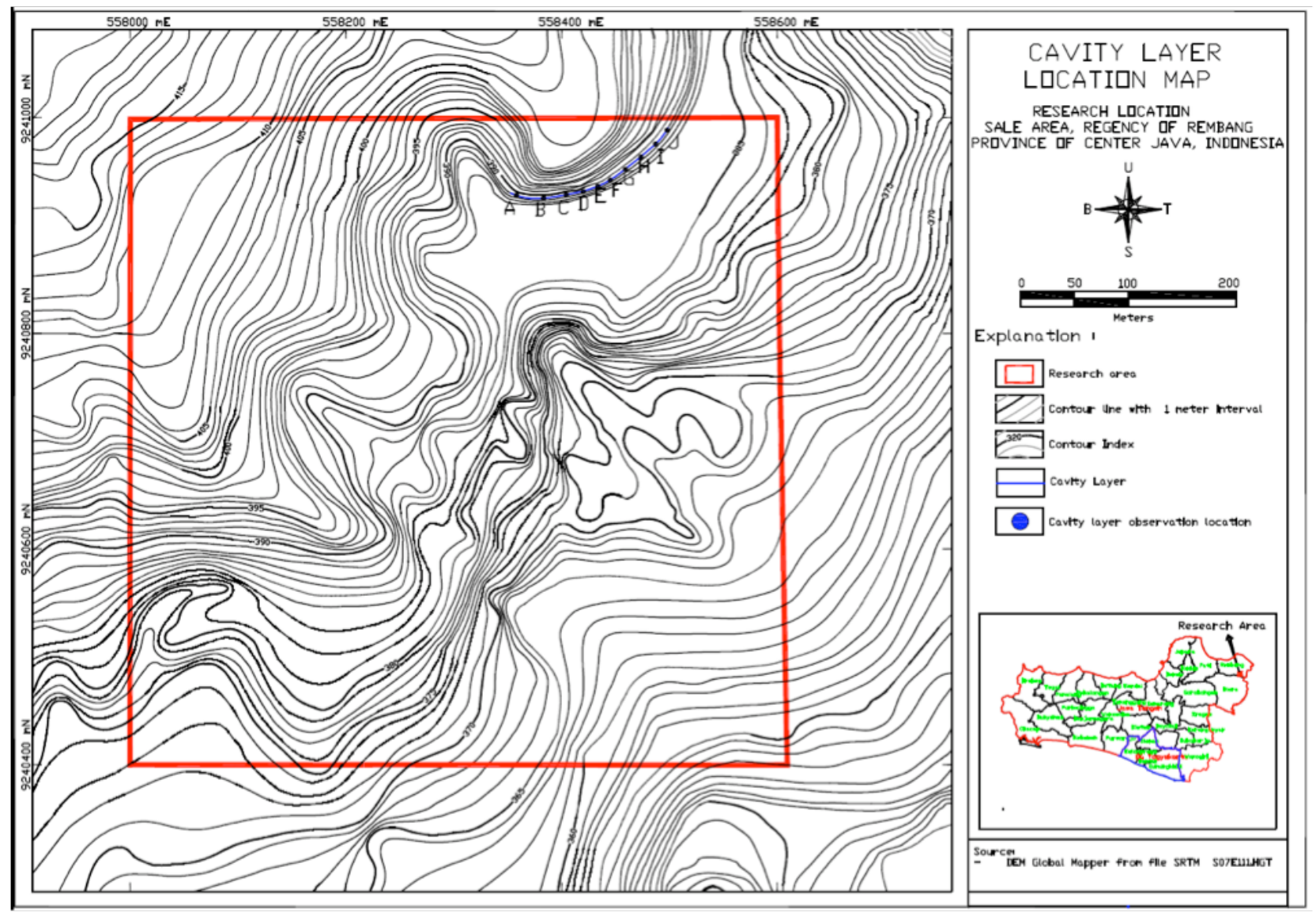

Figure 5: Cavity layer map. 


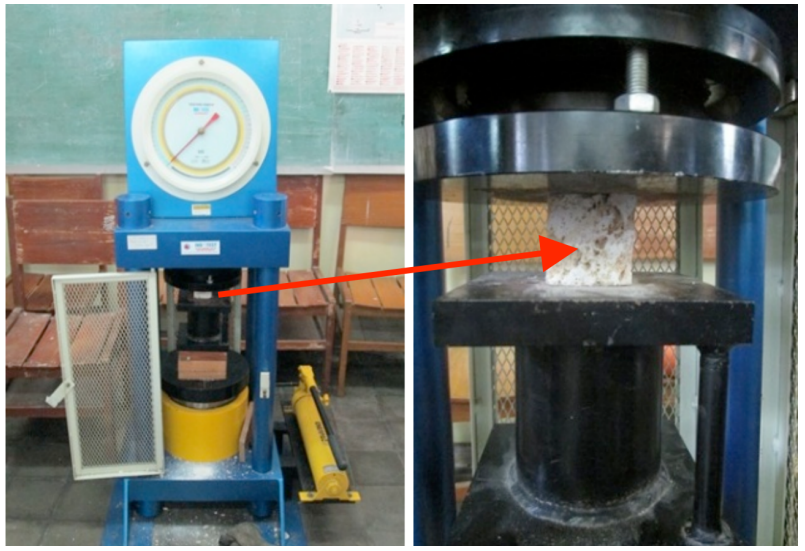

Figure 6: Uniaxial Compressive Strength (UCS) equipment.

\section{RMR estimation for rock mass quality of cavity zone in limestone quarry}

Identification of rock mass characteristic has been proposed to determine rock mass quality. This system provides a method for estimating the reduction in rock mass strength for different geological conditions as identified by field observations. Classification of rock mass quality based on the RMR, combined 5 (five) main parameters, i.e. strength of intact rock, RQD, spacing of discontinuities, condition of discontinuities and groundwater. Based on outcrop observations in limestone quarry area, all structure condition of the cavity limestone layer are described by the term disintegrated as the rock masses are poorly interlocked, heavily broken rock mass with a mixture or angular and rounded rock pieces (see Figure 4). Based on the uniaxial compressive strength (UCS) test, they have 8-12,4 MPa rock strength (Table 3 and Figure 6). Based on field observations, most of structure and surface conditions are very small block, rough rock surface (Table 4), moderately weathered to weathered (Table 5) and soft infilling material (Table 6). Condition of groundwater is dry (Table 7). The scores of RMR estimation for rock mass strength of cavity layer zone in limestone quarry are between 40 and 46. Estimation of the RMR is described in Table 8 .

\section{Conclusions}

The cavity limestone layer in the study area consist of very small blocks with rough surface, moderately weathered to weathered and soft infilling materials. The scores of RMR estimation for rock mass quality of cavity layer zone in the limestone quarry are between 40 and 46, indicating a poor rock mass quality. The cavity layer zone is a weak zone which has caused bench failures in the limestone quarry area.

\section{Acknowledgment}

Authors would like to thank to Management of PT. Sinar Asia Fortuna for the permit and facility provided during the research in the limestone quarry.

\section{References}

Bieniawski, Z. T. (1989) Engineering Rock Mass Classification, John Wiley dan Sons, Inc., Canada

Deere D.U. and Deere, D.W. (1967) Uniaxial Compressive Strength (UCS) after 20 years, Report manual, Contract DACW39-86-M4273, Department of the Army, U.S. Corps of Engineers, Washington DC.

Hustrulid, W. and Kuchta, M. (1995) Open Pit Mine Planning and Design, Volume 1 Fundamental, A.A. Balkema, Roterdam, Brookfield.

Situmorang, R. L., Smit, R., dan Van Vessem, E. J. (1992) Peta Geologi Lembar Jatirogo, Jawa, 1509 - 2, Skala 1:100.000, Pusat Penelitian dan Pengembangan Geologi, Badan Geologi, Departemen Energi dan Sumberdaya Mineral, Bandung.

PT. Sinar Asia Fortuna, 2013, Rembang Limestone Quarry, Final Report, Rembang, Jawa Tengah. 
Table 3: Uniaxial Compressive Strength (UCS) test.

\begin{tabular}{ccccccccccc}
\hline Parameters & \multicolumn{10}{c}{ Sample Code } \\
\cline { 2 - 12 } & A & B & C & D & E & F & G & H & I & J \\
\cline { 2 - 11 } Force $(\mathrm{kN})$ & 30 & 24 & 25 & 25 & 20 & 22 & 31 & 30 & 22 & 31 \\
\hline Area (sqcm) & 25 & 25 & 25 & 25 & 25 & 25 & 25 & 25 & 25 & 25 \\
\hline UCS (MPa) & 12 & 9.6 & 10 & 10 & 8 & 8.8 & 12.4 & 12 & 8.8 & 12.4 \\
\hline
\end{tabular}

Table 4: RQD score.

\begin{tabular}{cclcccc}
\hline No & $\begin{array}{c}\text { Sample } \\
\text { Code }\end{array}$ & Lithology & $\begin{array}{c}\text { Spacing of } \\
\text { discontinuity }(\mathrm{m})\end{array}$ & Jv & $\begin{array}{c}\text { RQD } \\
(\%)\end{array}$ & Score \\
\hline 1 & $\mathrm{~A}$ & Limestone & 0.027 & 37.03 & 0 & 3 \\
2 & $\mathrm{~B}$ & Limestone & 0.022 & 45.45 & 0 & 3 \\
3 & $\mathrm{C}$ & Limestone & 0.027 & 37.03 & 0 & 3 \\
4 & $\mathrm{D}$ & Limestone & 0.025 & 40.00 & 0 & 3 \\
5 & $\mathrm{E}$ & Limestone & 0.019 & 52.63 & 0 & 3 \\
6 & $\mathrm{~F}$ & Limestone & 0.019 & 52.63 & 0 & 3 \\
7 & $\mathrm{G}$ & Limestone & 0.032 & 31.25 & 11.87 & 3 \\
8 & $\mathrm{H}$ & Limestone & 0.027 & 37.03 & 0 & 3 \\
9 & $\mathrm{I}$ & Limestone & 0.019 & 52.63 & 0 & 3 \\
10 & $\mathrm{~J}$ & Limestone & 0.032 & 31.25 & 11.87 & 3 \\
\hline
\end{tabular}

Table 5: Spacing of discontinuity score.

\begin{tabular}{ccccc}
\hline No & $\begin{array}{c}\text { Sample } \\
\text { code }\end{array}$ & Lithology & $\begin{array}{c}\text { Spacing of } \\
\text { discontinuity }(\mathrm{m})\end{array}$ & Score \\
\hline 1 & $\mathrm{~A}$ & Limestone & 0.027 & 5 \\
2 & $\mathrm{~B}$ & Limestone & 0.022 & 5 \\
3 & $\mathrm{C}$ & Limestone & 0.027 & 5 \\
4 & $\mathrm{D}$ & Limestone & 0.025 & 5 \\
5 & $\mathrm{E}$ & Limestone & 0.019 & 5 \\
6 & $\mathrm{~F}$ & Limestone & 0.019 & 5 \\
7 & $\mathrm{G}$ & Limestone & 0.038 & 5 \\
8 & $\mathrm{H}$ & Limestone & 0.027 & 5 \\
9 & $\mathrm{I}$ & Limestone & 0.019 & 5 \\
10 & $\mathrm{~J}$ & Limestone & 0.032 & 5 \\
\hline
\end{tabular}


Table 6: Condition of discontinuity score.

\begin{tabular}{|c|c|c|c|c|c|c|}
\hline $\begin{array}{l}\text { Sample } \\
\text { Code }\end{array}$ & $\begin{array}{l}\text { Persistence } \\
(\mathrm{m})\end{array}$ & $\begin{array}{l}\text { Aperture } \\
(\mathrm{mm})\end{array}$ & Roughness & Infilling & Weathering & Score \\
\hline A & 2.55 & 1.4 & Rough & $\begin{array}{l}\text { Soft filling } \\
(<5 \mathrm{~mm})\end{array}$ & $\begin{array}{l}\text { Moderately } \\
\text { weathered }\end{array}$ & \\
\hline Score & 4 & 1 & 5 & 2 & 5 & 17 \\
\hline B & 2.78 & 1.5 & Rough & $\begin{array}{c}\text { Soft filling } \\
(<5 \mathrm{~mm})\end{array}$ & Weathered & \\
\hline Score & 4 & 1 & 5 & 2 & 3 & 15 \\
\hline $\mathrm{C}$ & 2.63 & 1.6 & Rough & $\begin{array}{l}\text { Soft filling } \\
(<5 \mathrm{~mm})\end{array}$ & Weathered & \\
\hline Score & 4 & 1 & 5 & 2 & 3 & 15 \\
\hline $\mathrm{D}$ & 2.48 & 1.4 & Rough & $\begin{array}{l}\text { Soft filling } \\
(<5 \mathrm{~mm})\end{array}$ & Weathered & \\
\hline Score & 4 & 1 & 5 & 2 & 3 & 15 \\
\hline $\mathrm{E}$ & 2.25 & 1.2 & Rough & $\begin{array}{l}\text { Soft filling } \\
(<5 \mathrm{~mm})\end{array}$ & Weathered & \\
\hline Score & 4 & 1 & 5 & 2 & 3 & 15 \\
\hline $\mathrm{F}$ & 1.95 & 1.3 & Rough & $\begin{array}{l}\text { Soft filling } \\
(<5 \mathrm{~mm})\end{array}$ & Weathered & \\
\hline Score & 4 & 1 & 5 & 2 & 3 & 15 \\
\hline G & 1.84 & 1.7 & Rough & $\begin{array}{l}\text { Soft filling } \\
(<5 \mathrm{~mm})\end{array}$ & $\begin{array}{c}\text { Moderately } \\
\text { weathered }\end{array}$ & \\
\hline Score & 4 & 1 & 5 & 2 & 5 & 17 \\
\hline $\mathrm{H}$ & 1.65 & 1.6 & Rough & $\begin{array}{l}\text { Soft filling } \\
(<5 \mathrm{~mm})\end{array}$ & $\begin{array}{c}\text { Moderately } \\
\text { weathered }\end{array}$ & \\
\hline Score & 4 & 1 & 5 & 2 & 5 & 17 \\
\hline I & 1.77 & 1.8 & Rough & $\begin{array}{l}\text { Soft filling } \\
(<5 \mathrm{~mm})\end{array}$ & Weathered & \\
\hline Score & 4 & 1 & 5 & 2 & 3 & 15 \\
\hline $\mathrm{J}$ & 1.58 & 1.5 & Rough & $\begin{array}{l}\text { Soft filling } \\
(<5 \mathrm{~mm})\end{array}$ & $\begin{array}{l}\text { Moderately } \\
\text { weathered }\end{array}$ & \\
\hline Score & 4 & 1 & 5 & 2 & 5 & 17 \\
\hline
\end{tabular}


Table 7: Groundwater score.

\begin{tabular}{ccccc}
\hline No & $\begin{array}{c}\text { Sample } \\
\text { Code }\end{array}$ & Lithology & $\begin{array}{c}\text { Condition of } \\
\text { Groundwater }\end{array}$ & Score \\
\hline 1 & A & Limestone & Dry & 15 \\
2 & B & Limestone & Dry & 15 \\
3 & C & Limestone & Dry & 15 \\
4 & D & Limestone & Dry & 15 \\
5 & E & Limestone & Dry & 15 \\
6 & F & Limestone & Dry & 15 \\
7 & G & Limestone & Dry & 15 \\
8 & H & Limestone & Dry & 15 \\
9 & I & Limestone & Dry & 15 \\
10 & J & Limestone & Dry & 15 \\
\hline
\end{tabular}

Table 8: RMR estimation of cavity layer zone in limestone quarry.

\begin{tabular}{|c|c|c|c|c|c|c|c|c|c|}
\hline No & 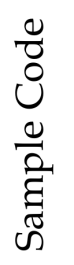 & Lithology & صٌ & $\widehat{\partial}$ & 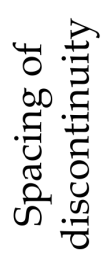 & 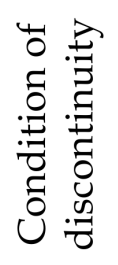 & 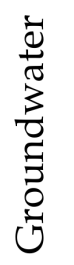 & 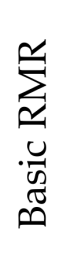 & 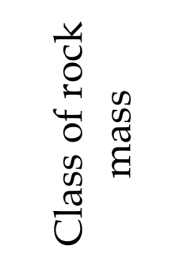 \\
\hline 1 & $\mathrm{~A}$ & Limestone & 2 & 3 & 5 & 17 & 15 & 42 & III (fair) \\
\hline 2 & B & Limestone & 2 & 3 & 5 & 15 & 15 & 40 & IV (poor) \\
\hline 3 & $\mathrm{C}$ & Limestone & 2 & 3 & 5 & 15 & 15 & 40 & IV (poor) \\
\hline 4 & $\mathrm{D}$ & Limestone & 2 & 3 & 5 & 15 & 15 & 40 & IV (poor) \\
\hline 5 & $\mathrm{E}$ & Limestone & 2 & 3 & 5 & 15 & 15 & 40 & IV (poor) \\
\hline 6 & $\mathrm{~F}$ & Limestone & 2 & 3 & 5 & 15 & 15 & 40 & IV (poor) \\
\hline 7 & G & Limestone & 2 & 3 & 5 & 17 & 15 & 42 & III (fair) \\
\hline 8 & $\mathrm{H}$ & Limestone & 2 & 3 & 5 & 17 & 15 & 42 & III (fair) \\
\hline 9 & I & Limestone & 2 & 3 & 5 & 15 & 15 & 40 & IV (poor) \\
\hline 10 & $\mathrm{~J}$ & Limestone & 2 & 3 & 5 & 21 & 15 & 46 & III (fair) \\
\hline
\end{tabular}

